\title{
VESTIR PALACIO A LA MODA Carlos III y el amueblamiento textil del Palacio Real de Madrid
}

POR

\author{
JOSÉ LUIS SANCHO
}

Patrimonio Nacional

\begin{abstract}
Charles III wanted in a 1763 wholly splendid decoration for his New Royal Palace in Madrid. The silk textures of the walls directed by count Gazzola, implied first a very important command to the Lions manufactures, but finally the Spanish ones at Valencia provided the most of all the furbishing, based on the french desings, white chinese "pekins» and Italian velvets put its touch of taste and exotism in this curious ensemble of fashion art, luxury and significant royal patronage of the home industry.
\end{abstract}

Si una de las primeras obligaciones del cortesano es comparecer en «traje decente» ante el Rey, como el Petimetre de serio que lleva camino à Palacio en la colección de trajes de Madrid, por la misma regla el Palacio Real tenía que estar también vestido a la moda. El ornato textil, las colgaduras de seda en las paredes, se desvanece en la memoria dentro del magnífico efecto general del conjunto, donde se imponen otros piezas con mayor peso específico, como las pinturas o los tapices, pero los tejidos reflejan sin embargo, por su mismo carácter movible y su limitada perdurabilidad, lo más característico de un momento del gusto. El Palacio Real de Madrid está tan marcado en su conjunto por el primer monarca que lo habitó, Carlos III, que las colgaduras escogidas para aquel estreno como vivienda regia resultan especialmente significativas, comparadas con las que en los dos siglos siguientes las han ido sucediendo; pero, además, el criterio general que rigió aquel amueblamiento es bien curioso por las relaciones que plantea entre el gusto y la moda, y también entre estos aspectos y las relaciones comerciales y la protección estatal hacia las manufacturas nacionales.

En la fecunda relación de la Corona de España con las sederías lionesas suele monopolizar la atención el reinado de Carlos IV. Por ello, las noticias sobre un amplio encargo de Carlos III ofrecen un especial interés, pero deben entenderse en un contexto general que aquí me veo obligado a resumir, remitiéndome a otras publicaciones donde he ofrecido otros aspectos sobre la decoración del Palacio Real Nuevo madrileño ${ }^{1}$.

\footnotetext{
1 Este trabajo forma parte de una investigación sobre el Palacio Real que desde hace años llevo a cabo en el Patrimonio Nacional. Algunos de los aspectos aquí tratados ya los adelanté en «Francisco Sabatini y el conde Gazzola: rococó y motivos chinescos en los Palacios Reales», Reales Sitios, n. ${ }^{\circ} 117$ (1993), pp. 17-26; el plano de la distribución durante el reinado de Carlos III se publicó en el número 109 (1991), «La planta principal del Palacio Real de Madrid», pp. 18-26, así como en la Guía de visita publicada por Patrimonio Nacional y Aldeasa, en sucesivas ediciones a partir de 1995. La copio-
} 


\section{Carácter, gusto y moda: adjudicar a cada sala su tela}

Cada sala de Palacio tiene una función, que define su carácter, y este marca su ornato. Esto se advierte contrastando la planta del piso principal de Palacio con las noticias aquí aportadas. Por otra parte, el gusto de la época está marcado por las influencias externas, que dictan una preponderancia de los diseños franceses, pero también la atención a ciertas especialidades italianas, y una tendencia muy general a la chinoiserie propia del tardo rococó que es la moda vigente para Carlos III. Las sedas francesas, los brocados y terciopelos italianos y los pequines chinos se armonizan, sirviendo en unos u otros espacios en función de su carácter. Quien rige su disposición, el sumo sacerdote del gusto en Palacio para el amueblamiento textil, es un hombre de confianza de Carlos III, el conde Felice Gazzola ${ }^{2}$; con orgullo él mismo expuso «... que en Nápoles [el rey] se dignó emplearme en muchas dependencias de esta naturaleza, y lo propio la gloriosa memoria de la Reina...» ${ }^{3}$. Aguerrido militar, director del Real Colegio de Artillería de Segovia, hombre culto, Gazzola es un hombre de su siglo, y a su sentido del gusto no escapa la atención a las novedades de la moda. Dictada por Francia, a través de Lyon como manufactura y de Versalles como difusor, la moda ha de imponerse también en las sedas para las paredes del Palacio Real.

Gazzola impuso la moda francesa en el conjunto del Palacio, encargando modelos lioneses, pero poniendo, al fin, sedas valencianas. Optó sin embargo por ricos brocados y terciopelos italianos para dos piezas de especial significado simbólico: el Salón del Trono, y el dormitorio de los recién casados príncipes de Asturias; es decir, la representación de la Soberanía, y su germen de futuro. A esta preferencia por las formas y la materia de Italia no debían ser ajenos el propio rey y su familia, apenas llegados de Nápoles y, en el caso de la princesa María Luisa, de Parma. Por otra parte, en las habitaciones más íntimas dentro de cada uno de los cuartos de las personas reales se imponían, según el gusto de la época, las telas chinescas.

El proceso de selección de telas, y su fabricación o compra, se desarrollaron a lo largo de 1763 y del año siguiente, acelerándose para llegar a tiempo al primero de diciembre de 1764, fecha marcada por el Rey para comenzar a residir en su Palacio a la vuelta de la «jornada» de El Escorial. Para mayor claridad, es preferible presentar los tejidos por procedencias.

\section{Las sedas de Lyon}

En marzo de 1763 se pidieron a la manufactura lionesa de Caillat \& Chirac «muestras en grande, sin oro ni plata, de estofas para colgaduras de verano e invierno para cuartos grandes y gabinetes del último buen gusto y moda, (y) que enviasen sus precios» ${ }^{4}$. Los sederos franceses

\footnotetext{
sa documentación sobre este asunto aquí citada se encuentra en el Archivo General de Palacio, fondo de «Obras de Palacio», leg. 442. Complementarios de este texto son otros dos artículos basados en las mismas fuentes, y que ahora aparecen: «El Salón del Trono en el Palacio Real de Madrid», en Antologia di Belle Arti, n. ${ }^{\circ}$ 59-62, Allemandi, Turín, 2000, pp. 85-106, y «Las sedas de Valencia encargadas por Carlos III para el Palacio Real de Madrid», Archivo de Arte Valenciano, año LXXX, Valencia, 1999, pp. 72-79, donde cito toda la bibliografía sobre este asunto, que juzgo preferible no repetir aquí. Agradezco cordialmente a Alvar González-Palacios sus amistosos consejos.

${ }^{2}$ Me remito a «Sabatini, Gazola...» cit. en n.1, donde se recoge la bibliografía sobre este peculiar personaje, en especial las concienzudas investigaciones de Pérez Villanueva.

3 Gazola a Muzquiz, 21.5.1766.

${ }^{4}$ Sólo una mención marginal sobre Caillat \& Chirac se encuentra en la obra de referencia básica, hasta el momento, sobre las manufacturas lionesas en el periodo: Soieries de Lyon. Commandes royales au XVIIIe siècle (1730-1800). Catálogo de la exposición dirigida por Pierre Arizzoli-Clémentel y Chantal Gastinel-Coural, Musée Historique des Tissus, Lyon 1988; de especial interés en este volumen son las «notes et documents» presentados por Mme. Gastinel-Coural (pp. 28103), con referencias prácticamente nulas sobre Carlos III, pero abundantísimas al periodo de Carlos IV, a cuyo respecto la obra clásica es la de Alexandre Poidebard y Jacques Chatel, Camille Pernon, fabricant de soieries a Lyon... 1753-1808, Lyon 1912. Sobre el gusto de la familia real española, que Caillat \& Chirac empezaron a tentar, y también sobre el de la
} 
obedecieron con presteza bien comprensible y veinte días despues, el seis de Abril, ya habían llegado las primeras muestras, a las que una seman más tarde se sumaron otras que podemos imaginar de modo bastante satisfactorio merced a sus memorias explicativas, que reproduzco en apéndice por su extraordinario interés. Queda para los especialistas en historia de los textiles el identificar las piezas de este muestrario con las que se puedan conservar en otros lugares y particularmente en el Musée des tissus de Lyon, ya que buena parte de ellas se habían tejido previamente con otros destinos, entre los que destaca el Palacio de Versalles ${ }^{5}$.

Tales géneros, decían Caillat \& Chirac, sortent d'une fabrique unique dans le genre qu'elle travaille, outre le goût et la nouveauté ce qui est peint est d'une solidité a toute épreuve. Pensaban enviar pronto muestras de género y gusto distinto, et particuliérement ceux de differents meubles qui sont dans les appartements du Roy à Versailles, et dans lesquelles en y faisant quelques légers changements les meubles seront superbes par la richesse de bordures, et n'auront rien de commun avec ceux de Versailles parce que les bordures peuvent aller sur toutes sortes de fonds.

Advertían, por otra parte, que las muestras «habían sido manoseadas perdiendo la frescura de una tela recién fabricada, y así no hay que considerarlos que por el efecto que puedan hacer. (Además) se pueden cambiar los fondos de las colgaduras por otros dibujos y colores, según se ordene». Así, y pese a las especificaciones hechas, enviaron una muestra de terciopelo con dorado sólo para mostrar el dibujo. El 23 de abril y el 7 de mayo remitieron dos muestras de excepcional originalidad y riqueza: la primera de estas telas, calificada como «soberbia» y «pieza única» había sido realizada en bordado a mano — con el fin de que pudiera ser enseñada cuanto antes - pero aseguraban que ganaría mucho tejida en telar. El precio de este modelo podía variar según la materia, pero en cualquier caso sería caro, al tener que hacerse el telar $e x$ profeso; indicaba como destino el realizar una cama de parada completa, y podría tener aspecto muy diferente según el fondo que se decidiese darle. La segunda muestra, bordada también a mano, fue enviada algo más tarde, pero junto con la primera los fabricantes lioneses mandaban también otra de fantasia y un paquete con otras cinco de «taffetas chiné» para dos conjuntos decorativos o «muebles», calificados como «extraordinariamente bellos, claros, y que producirán un magnífico efecto en tapicerías» ${ }^{6}$. No se ha conservado lista de las enviadas en mayo ${ }^{7}$.

aristocracia madrileña, es interesantísima, y aún no explotada como merece, la correspondencia entre Grognard y Peyron citada por Poidebard \& Chatel, pp. 8-12, y conservada en la Bibliothèque de la Ville de Lyon, ms. 1923. Agradezco con cariño a Marga Torrione su consulta.

${ }^{5}$ Entre las muestras que custodia el Musée Historique des Tissus, cuyo inventario corre a cargo de Marie-Jo de Chaignon, seguramente podría identificarse más de una de las mencionadas en las listas de Caillat \& Chirac, pero me ha sido imposible dedicar tiempo y esfuerzos a tal repaso sobre el terreno.

En la obra de Marie Bouzard, La soierie lyonnaise du XVIII au XXe siècle dans les collections du Musée des Tissus de Lyon. Editions lyonnaises d'art et d'histoire, 1997, algunos de los tejidos mostrados en las dos primeras decenas del catálogo se aproximan a los descritos por Caillat \& Chirac. Por ejemplo, los tafetanes de los números 1 , 4 y 7 se asemejan al n. ${ }^{\circ} 13$ del catálogo.

${ }^{6}$ Caillat \& Chirac a Squilace, 23 de abril de 1763: «Excellence. Mr. De Clerac capitaine d'artillerie... emporte un paquet a l'adresse de V.E., ou est un échantillon d'un meuble superbe dont on a fait faire le dessin exprés, et que nous avons fait executer en broderie pour en faire voir tout l'effet, et pour l'avoir beaucoup promptement que s'il fût eté fait sur le métier; il rendra encore davantage fabriqué sur le metier, que par la broderie, c'est un morceau unique, et dont vous avez la premiére vue. Le meuble peut s'executer dans le fonds qui plaira mais selon le fond il faudra peut être en changer les nuances; il y a sur l'étoffe un mémoire attaché, de differents prix auxquels l'échantillon peut s'executer selon les differentes genres d'étoffe dans laquelle l'on voudra l'executer. Les fabriquants peuvent executer les fonds, ciel de lit et couverture d' un seul dessein, ces morceaux deviendront plus chers à cause des metiers qu'il faudra faire exprés, alors ce sera un meuble superbe et comme il n'y en a point.

Les mêmes fabriquants travaillent encore un meuble dans un autre genre qui fera encore plus beau et plus frappant, malgré qu'il y aie nombre de brodeuses qui l'executent, nous n'avons pu l'avoir pour profiter de l'occasion et du depôt de mr. De Clerac, mais si tôt que sera fini ce qui menera environ à quinze jours, nous l'enverrerons à V.E., nous la prions de ne poin prendre de determination que ce dernier échantillon ne le soit parvenu. 
Temiendo las imitaciones, los fabricantes insistían para que no fuesen enseñadas y que se les devolviesen ${ }^{8}$. Tal devolución se llevó a cabo en agosto, con la excepción de las ocho muestras escogidas por el rey, «diciéndoles que ejecutaré lo propio con éstos siempre que sus dueños no quieran aguardar que el rey tome su última resolución; y que en tanto no tengan recelo ninguno de que se saque copia de ellas, ni que se hagan ver a nadie».

Las sederías elegidas por el rey fueron: «N. ${ }^{\circ} 1$, un corte de terciopelo de ana y media - con el fondo en oro-; . $^{\circ} 1$, otro de canelé fondo carmesí floreado con chenilla, cordonete y cañutillo; n. $^{\circ} 2$, estofa a dos borduras grandes en forma de enrejados; n. $^{\circ} 2$, id. a borduras chicas; $\mathrm{n}^{\circ} 7$, grodetur con flores y figuras; . $^{\circ} 5$, tafetán pintado con flores a la chinesca; ${ }^{\circ}{ }^{\circ} 1$, moer con aguas pintado con flores a la chinesca, y compuesto de tres paños; $n .^{\circ} 8$, grodetur floreado con seda dorada, y con figuras» ${ }^{9}$.

Il y a aussi un petit echantillon de fantaisie avec un mémoire qui y est attaché, de même qu'un paquet qui contient cinq échantillons de taffetas chiné pour deux meubles dont les desseins sont extremmement beaux, bien nets et qui doivent produire un bien grand effet en tapisseries, le mémoire est joint au paquet.

Nous avons eu avis de Paris que les fréres Le Couteulx nos amis avaient expedié à V.E. ce que nous leurs avions adressés, en recommandant à l'amis de Bayonne de remettre le tout au courier d'Espagne.

Nous renouvellons à V.E. qu'elle doit faire moins d'attention au fond des échantillons qu'à leur dessein parece que nous donnerons tous nos soins pour la plus parfaite execution.

Nous prions encore V.E. de nous envoyer exactement tous les échantillons quand elle aura pris touttes ses déterminations parce que nous avons été obligés de prendre des engagements de les raporter aux fabriques qui nous les ont confiés. Mais rien ne presse, et elle a le temps de se decider a son aise, nous l'assurons toujours que nous nous employerons avec toutte l'exactitude possible à remplir ses vues et ses ordres et nous ne cesserons d'être avec un respect infini, etc.

${ }^{7}$ Caillat y Chirac a Squilace, 7 de mayo de 1763: Excellence: nous expérons que vous avez actuellement reçu non seulement nos premiers envoys, mais encore celui que nous vous avons fait par mr. De Clerac capitaine d'artillerie, nous l'aprendrons avec plaisir. Actuellement nous faisons encaisser le superbe échantillon que nous avons fait faire, qui est au delá de tout ce qui nous avons envoyés a V.E., aussi esperons nous qu'il rencontrera toutte la satisfaction. Le prix en est bien haut mais il y a un ouvrage inmense et l'execution de cette étoffe en meubles sera de la derniére richesse sans qu'il y ait ni or ni argent, comme le dessein de cet échantillon de même que du precedent que vous a porté mr. De Clerac et leur execution coutent aux fabriquants des peines et des frais bien considerables, nous suplions V.E. de ne pas permettre que ces desseins soient (copiés?), et soit qu'ils conviennent ou ne conviennent pas elle aura la bonté de nous les faire renvoyer sûrement et dans les mêmes caisses s'il est possible parce que à cause de la chenille il ne faut pas qu'ils soient presses. S'ils conviennent, les fabriquants pourront faire les fonds du lit, des pentes et de tous les meubles du même dessein et de la largeur necessaire, il faudra pour cela envoyer de mesures bien exactes de même que des tapisseries pour les hauteurs et largeurs, pour travailler sûrement, il faudroit faire tendre les appartements de quelques toiles grossieres et de bas prix sur chacune desquelles ce qui est hauteur et ce qui est largeur seroit marqué à fin qu'on le connut ici, et que l'on puisse en consequence travailler les étoffes de la largeur convenue. Il faudroit faire des mêmes détails i l'on les vouloit d'un seul dessein pour les dedans du lit ainsi que des dessus des chaises et fauteils; vous nous envoyerez alors ces toiles et les meubles pourraient alors être envoyés pour être tendus c'est à dire les tapisseries, ou tellement ajustés qu'il n'y auroit qu'à unir les hauteurs destinés pour chaque largeur de tapisseries, dessus des portes et entre-deus des fenêtres.

Il y a joint a ce bel échantillon, un nouveau qui nous a eté remis et que peut bien faire.

La tapisserie de toille serviroit aussi pour faire les meubles d'été, c'est à dire pour servir de mesure, il nous parait que l'envoyé de la toille est le seul expedient qui puisse être le plus utile pour bien faire, alors comme l'avons dit de votre excellence il ne faut pas omettre d'y notter tout ce que peut servir a connoître les hauteurs et que y chaque partie sera destinée en metant sur chacune un numero, et faire un paquet separé de ce qui servira pour chaque salle ou cabinet. Nous répetons à V.E. que nous avons mis tout notre zéle et toute notre activité pour qu'elle aie lieu d'être satisfaite et l'engager a nous continuer sa bienveillance. ... \&c.

${ }^{8}$ Cartas de 6 y 9 de Julio de 1763. Anunciaban igualmente la repercusión del aumento del precio de la seda.

9 Esta nota debe haber sido redactada por Gazzola, o por Le Goujeux:

«Notte des échantillons qu'on a choisi, et retenu jusqu'à ce que S.M. aye prise sa derniére resolution.

N. ${ }^{\circ} 1$, un coupe de velours de $11 / 2$ aune avec le fond en or.

n. ${ }^{\circ} 1$, autre de canelé fond cremoisi à ramages, avec chenille, cordonet, et canutille.

n. ${ }^{\circ}$, autre de etoffe à deux grandes bordures à trillage

$n .^{\circ}$, autre ditto. à quatre petites bourdures

n. 7 , autre de grodetour à ramages, et figures

n. 8 , autre ditto

$\mathrm{n} .^{\circ} 5$, autre de taffetas peint à la chinoise

$\mathrm{n} .^{\circ} 1$, autre d'amuer ondé peint à la chinoise de trois toils.

Le reste des échantillons remis, on les renvoit a mr. Calliat \& Chirac par le canal de mres. de La Borde \& Nogué de Bayonne. 
Ante sus reiteradas quejas, se les devolvieron las muestras el 7 de septiembre de $1763^{10}$; simultáneamente, Caillat y Chirac escribían a España informando que el fabricante de tafetanes y moirés à la chinoise se ofrecía a ir a Madrid, pero la balanza ya se había inclinado a favor de las fábricas españolas e italianas. Il est triste pour nous qu'il n'aie été prise aucune determination pour ces meubles et que les soins que nous avions pris pour répondre à vos ordres aient été inutiles ${ }^{11}$.

\section{El gran encargo a Valencia}

La suspensión brusca y total del encargo a Lyon mientras se pedía a las fábricas valencianas que fabricasen prácticamente todas las colgaduras para Palacio, y que aún se diesen largas a Caillat \& Chirac cuando al intendente de Valencia ya se le habían precisado los colores y texturas y, lo que es más significativo, se le habían enviado los dibujos, son hechos que hablan por sí mismos. En honor a la verdad, no es la misma actuación de esta naturaleza documentada en la Corte de España durante aquellos años ${ }^{12}$. En otro lugar me he ocupado del encargo a Valencia con la extensión debida, y aquí es sólo preciso destacar la semejanza de colores y diseños entre las sedas propuestas por Lyon, y las finalmente labradas a toda velo-

\footnotetext{
à St. Ildefonce le 22 aoust 1763".

«Muestras de telas de Lion, que ha escogido el Rey.

$+\mathrm{N} .^{\circ}$, un corte en terciopelo de una ana y media.

n. ${ }^{\circ} 1$, otro de canelé fondo carmesí floreado con chenilla, cordonete y cañutillo.

$+n .^{\circ} 2$, estofa a dos borduras grandes en forma de enrejados.

$+\mathrm{n} .^{\circ} 2$, id. a borduras chicas

$+\mathrm{n} .^{\circ} 7$, grodetur con flores y figuras

n. ${ }^{\circ} 5$, tafetan pintado con flores a la chinesca.

$+\mathrm{n} .^{\circ} 1$, moer con aguas pintado con flores a la chinesca, y compuesto de tres paños

$+\mathrm{n} .{ }^{\circ} 8$, grodetur floreado con seda dorada, y con figuras.»

10 «Excellence: Depuis la lettre que V.E. prit la peine de nous écrire le 11 du mois passé, nous avons toujours attendu de semaine en semaine d' apprendre sa dernière determination sur tous les échantillons de nos fabriquies que nous avons eu l'honneur de lui envoyer, ou soit leur renvoy suivant la prière que nous lui en avons faite par toutes nos precedentes; mais nous voyant privés de ses nouvelles nous venons la supplier instamment d'avoir la complaisance de nous renvoyer en réponse tous les mêmes échantillons, parce que les fabriquans qui nous les avoient confié et envers qui nous avions pris des engagements par écrit de les leurs rendre aprés l'espace de trois mois, n'ont cessé de nous solliciter et de nous presser jusqu' à present, mais dans l'inquietude et dans la crainte aù ils sont que leurs desseins ne soient copiés (surquoi nous avons tout employé pour les rassurer et les engager à prendre encore patience), ils nous ont menacé de se servir de la voye juridique pour nous forcer de les leurs rendre, en sorte que nous nous trouvons dans un labyrinthe qui nous chagrine d'autant plus que nous n'avons jamais manqué à nos engagements, et que l'exactitude doit être l'unique principe des negotiants. Nous reiterons donc toutes nos instances à V.E. de ne point perdre de temps à nous faire le renvoy de tous ces échantillons à fin de nous éviter des désagrements que nous sommes dans le cas d'essuyer. Si nous eussions pu prevoir que V.E. ne se decidât pas sitôt, nous nous serions arrangés ici en consequence, mais dans la lettre qu'elle nous fit l'honneur de nous écrire à cet égard, elle nous paroissoit si pressé que nous employâmes aussitôt tous nos soins pour la contenter soit en faisant le choix de tous les échantillons qui pouvoient convenir pour des meubles, et soit en faisant faire exprès de magnifiques dont le travail a été long, penible et couteux, enfin si V.E. a fait le choix de quelques uns pour nous en commettre nous donnerons tous nos soins pour remplir ses intentions, et c'est dans cette assurance que nous avons l'honneur de lui renouvelles tout nôtre zéle et le profond respect avec lesquels nous ne cesserons d'être... etc., Caillat \& Chirac. Lyon, le 31 éme Août $1763 »$.

"Caillat \& Chirac continuaron siendo proveedores del propio Squilace, o mejor dicho de su esposa para quien hicieron un rico traje (garniture) juzgado por la interesada du dernier bon goût, según carta a Squilace de 22 de febrero de 1764 , en la que pedían la devolución de las ultimas de sus muestras que todavía seguían en Madrid, y resuelta el 19 de marzo: «remitan este resto de muestras a LaBorde y Nogué de Bayona para que lo encaminen a Caillat \& Chirac, y dése a éstos el aviso relativo».

${ }_{12}$ Cfr. Amelia Aranda Huete, en Reales Sitios, 1998.

13 A.G.P., fondo «Obras de Palacio», leg. 442: Expediente sobre hilo de oro, con cuatro muestras del mismo, $1766-1767$. 18.12.1766, Gasparini a Muzquiz, pidiendo hilo de oro para la colgadura de la Cámara de Parada.

12.1.1767, Ventura de Llovera a Muzquiz, de París: que ha recibido las cuatro muestras de las que debe enviar hilo. 14.4.1767, recibo de Gasparini.
} 
cidad en Valencia. Los moarés parecen haber sido en particular del gusto de Carlos III, que se manifiesta también en el empeño con que Gazzola procuró obtener una imitación lo más perfecta posible del Bleu du Roi de Francia, del que se conservan en el Archivo de Palacio tres interesantes muestras de diferentes matices. Este celo en la perfección de la materia prima para el adorno textil no es único en Palacio, aunque aparece significativamente en los detalles de mayor importancia, como en la elección de calidad para el hilo de oro con destino a la colgadura bordada de la Cámara del Rey, o Salón de Gasparini; cuatro muestras se conservan con todo su brillo ${ }^{13}$.

La mayor parte de las salas de Palacio fueron colgadas con sedas valencianas, pues con ellas fueron colgadas las primeras y segundas antecámaras de todos los cuartos y buena parte de las piezas intermedias, reservando sólo de modo inequívoco al exotismo las más íntimas y reducidas pero numerosas, los gabinetes, y otorgando especial relevancia, mediante ricos tejidos italianos, a la Galería del Trono y al dormitorio de los príncipes.

\section{Los encargos a Italia}

El Salón del Trono recibió un ornato muy coherente bajo la directa supervisión de Gazzola, quien decidió sus características esenciales (incluída, al parecer, la elección de Tiepolo para pintar la bóveda) y encargó a su paisano G.B. Natali los diseños para los todos los elementos, desde los muebles hasta la cenefa bordada con hilos de plata sobredorada que recorre la colgadura de terciopelo, tejido en Génova ex profeso. La coherencia, valor y conservación de este conjunto decorativo obliga a tratar de las circunstancias de su encargo con tal extensión que aquí no cabe más que mencionarlo ${ }^{14}$.

La importancia simbólica de la sala del dormitorio de los Príncipes fue sin duda determinante para decidir amueblarla también con una colgadura italiana especialmente rica, pero no hay que olvidar que, estrenada en diciembre de 1764 la nueva residencia regia, en la primavera siguiente tenía lugar la boda del Príncipe Carlos con su prima Maria Luisa, y en tal coyuntura no puede extrañar un especial énfasis en las habitaciones de quienes encarnaban la perdurabilidad de la dinastía, ligando así la imagen del Nuevo Palacio con una renovación familiar. Italianas eran también las únicas pinturas de esta sala, las sobrepuertas realizadas en Nápoles por Fischietti. Sin más cuadros que la ocultasen lucía aquí una colgadura de terciopelo con fondo de oro, encargada a Venecia, por medio del embajador de la Serenísima, «pues quiere el Rey que despues de la suya sea la más magnífica» ${ }^{15}$. Una vez que Sabatini hubo transmitido las medidas del dormitorio del Príncipe a Gazola ${ }^{16}$, éste envió los diseños para esta tapicería y la del Trono a principios de noviembre de 1764, pero todavía no podía precisar el presupuesto exacto «por la razón de no haberse todavía dada la comisión entera para los modelos de cama, y dosel que no se han acabado por tomar las medidas.» ${ }^{17}$. Hasta marzo no envió Gazzola a Gé-

\footnotetext{
14 Ya ofrecí un breve resumen en «Sabatini y el conde Gazzola...», cit. en n. 1. Ahora se publica toda la documentación en Antologia di Belle Arti, 2000, cit. en nota 1.

15 Gazola a Squilace, 30 de abril de 1764.

16 Squilace a Sabatini, sobre las medidas del dormitorio del Príncipe, 7.11.1764.

17 Gazola a Squilace, 19 de noviembre de 1764, remitiéndole «dos paquetes que le suplico se sirva incaminar a su destino. Contiene diseños y instrucciones para las colgaduras de la sala del dosel y cámara de la cama de parada del Sr. Príncipe de Asturias.

Como naturalmente serán precisas anticipaciones de dinero a los que han entraprendido estas obras espero que V.E. se servirá mandar se franquee algún dinero por medio de D. Luis Beltrami al señor Jaime Gentile, caballero de Génova, y a subteniente D. Antonio Moscati en Napoles, no siéndome posible calcular las sumas precisas ni de Génova ni de Nápoles.. El gasto es de consideración, como V.E. no ignora, y me parece no sobrará dinero si dispondrá que por ahora se hallen en Génova treinta a cuarenta mil libras de aquella moneda, y doce mil ducados en Nápoles a la disposición de los enunciados sujetos».
} 
nova el diseño de la cama de parada del Príncipe ${ }^{18}$. Los paños de terciopelo que estaban encargados y los de raso que habían de servir para dicha cama fueron enviados de Génova a Barcelona en mayo ${ }^{19}$, y puesto que no vuelve a ser mencionada Venecia, cabe pensar que se había cambiado de idea y fabricado todo en la República del Tirreno; como se deja claro que se trata de la cama de aparato, y que con ella se relacionan las muestras de la colgadura de la misma sala, cabe la posibilidad de que la cama y colgadura encargadas a la Serenísima fueran las del dormitorio efectivo del Príncipe, pero carecemos de las pruebas necesarias para convertir en afirmación esta conjetura ${ }^{20}$. En cualquier caso, las colgaduras de Génova llegaron en julio ${ }^{21}$. Una carta del mayordomo mayor al primer arquitecto proporciona precisiones sobre la pasamanería: «En cuanto a decirme V.S. que habiéndose empezado a traer de Génova la colgadura de la cama del Príncipe, es preciso mandar fabricar una cantidad de fleco, borlas y cordón de plata para ella, soy de dictamen que, pues ha corrido dicha cama a cargo del sr. conde de Gazola, y que es muy regular que el todo del coste de ella sea comprendido bajo de una misma cuenta, V.S. se lo proponga al propio sr. conde de Gazola, a fin de que disponga el fleco, borlas y cordón, como le parezca más conveniente» ${ }^{22}$.

A la vez que el cuarto del Príncipe se preparaba el de la Princesa de Asturias, si bien con menor ostentación y gasto pues los tejidos no se encargaron a Italia sino a Talavera. Tres fueron al menos las colgaduras allí hechas. La de la Pieza de Parada, que ha de ser la del tocador, ya estaba entregada al director de aquella fábrica en febrero de 1765, y parece por tanto posible que se hiciese en Madrid bajo la inmediata supervisión de Gazzola. Por el contrario, la propia manufactura proveyó la traza para las sedas con destino a la antecámara, como informa una nota sin fecha de Gazola a Scellari: «Oggi è stato da me il direttore della fab. di Talavera che si chiama D. Giusepppe Gil della Torre con il disegno della stoffa che dovrebbe far tessere per l'anticamera della sigra. Principessa di Asturias. Se dunque il Sr. Marchese lo aprova potrá darci l'ordine corrispondente e per ció al piede le pongo la direzione, afinché non si atrassi il Rl. Servizio...» ${ }^{23}$. Por último, la más vistosa era, lógicamente, la de la sala de besamanos del cuarto «de grodetur color de plata, matizado en doble espolinado, y a doble liage», con cene-

18 Gazola a D. Antonio Scellari, Madrid 12 de marzo 1765: «La prego incaminare a Genova l'annessa per il sig. Giacomo $\mathrm{M}^{\mathrm{a}}$ Gentile. Contiene il disegno del letto destinato al quarto di parata del sig. Principe di Asturias, e siccome detto sig. Giacomo $\mathrm{M}^{\mathrm{a}}$ Gentile mi scrive che mi mandará certe mostre del parato, et inquadrature se non sarà per questo la prego scrivere il venturo a Belzan che ne riceva li due piccioli pachetti mandandoli collo straordinario di Napoli...»

19 Gazola a Squilace, 18 de mayo de 1765.

${ }^{20}$ Así parecen indicarlo los términos en los que se expresaba Gazola a Squilace el 18 de mayo de 1765: «Con lettera de 6 del corrente mi scrive il Sig. Giacomo $\mathrm{M}^{\mathrm{a}}$ Gentile da Genova che si lusinga d'avere pronta, et in termine tutta la comissione apogiatali delli veluti cremisi, e del rasone per la camera da letto del Sig. Principe di Asturias. Il Sig. Marchese Grimaldi si è contentato che a poco, a poco mi mandi ciò che corrisponde al letto per la premura di porlo in opera, ma con la stessa providenza non potrà spedirsi il resto per essere troppo voluminoso. In conseguenza mi dimando se dovrà spedirlo con falucca a posta a Barcelona quando non si presenti altra occasione sicura, e meno costosa.

Io non sono informato, ne devo esserlo del tempo che possa aversi per mobigliare et adattare il rasone del quarto del Sig. Principe di Asturias ne sono in caso di pigliare sopra di me una tale providenza come la sugerisce detto sig. Grimaldi e V.E. potrá darmi li suoi oracoli acció comunicati a detto cavagl. resterá servito il Padrone -el Rey- come desidera, e pronto a cenni di V.E. con il mio ossequio mi confermo, di V.E. ...»

${ }^{21}$ El 2 de julio llegaron siete cajones, seis de ellos con 23 piezas de terciopelo y el séptimo con rasón de plata. El día del del mismo arrivaron a Barcelona «cuatro cajas con el resto de las colgaduras de Génova para el Real Palacio».

22 Montealegre a Sabatini, 12.5.1765: «En la carta que recibo de V.S. por el parte de hoy veo quedar enterado, y en practicar todo lo que previene en la mía de antesdeayer, como de la resolución del rey en punto a colgaduras, que en el mismo día comunicó a V.S. de mi orden el jefe de la Rl. tapicería.» Sabatini a Squilace, 12.7.1765, sobre los diversos gastos de ornatos para la boda de los Príncipes: «... cordones y borlas de plata para la cama... que se han suplido por la consignación de la Fábrica, y pide se le libren separadamente por T.Mayor. Resuelto así por Squilace en la minuta, 19 del mismo mes.

${ }^{23}$ Gazzola a Squilace, 2 de Febrero de 1765: «Per il mobile della sala dell'aparato della Sigra. Principessa di Asturias si è concluso il disegno, e datte le misure al direttore della fabrica di Talavera onde non resta se non che V.E. si serva dar l'ordine corrispondente a chi spetta per l'esecuzione de 60 teli di stoffa che si necesitano, oltre la inquadratura come presentemente si usa in somiglianti camere di parata, e con tutto il rispetto ho l'onore di confermarmi, etc == Felice Gazola». Squilace dispuso que se hiciera, y que una vez hecho se pagase al precio ajustado con Gazola. 
fas «de grodetur color lila fino con oro, en diferentes hilados, y con matices de seda, uno y otro espolinado»; además se enriquecía con sobrepuestos, «treinta y siete flores de oro grandes en glasé... de media vara de largo, y dos tercias de ancho para cada una.» Las cortinas de invierno eran como la colgadura, pero las de verano eran de tafetán blanco ${ }^{24}$.

Todas estas sedas talaveranas no debieron servir hasta el invierno siguiente de 1765-66, pues no se pagaron hasta principios de este último año, a la vez que diversos géneros complementarios de seda y pasamanería ${ }^{25}$.

\section{Los gabinetes chinos}

El gusto por la chinoiserie propio del tardo rococó dominaba en los aposentos privados de las personas reales, de modo bien coherente, pero cuyo principal testimonio subsistente es la colgadura bordada de la Cámara de Carlos III. En otro momento ya quedaron expuestos los aspectos básicos de esta proliferación de «pequines», pero no hubo entonces lugar para exponer la rica documentación, que permitirá ligar algunos techos y elementos todavía conservados in situ con los «pequines» conservados en el almacén de telas y con otros conjuntos conservados en otros palacios, como los de la Residencia Real de Drottingholm, pues no en balde la Compañía Sueca jugó un papel esencial en el amueblamiento del Palacio de Madrid.

En su carta de 30 de abril de 1764 Gazola mencionaba, junto a los encargos de Italia y los mueres y damascos de Valencia para antecámaras con cuadros, cinco gabinetes chinescos que habían de ser pintados por un artista que había designado Squilace — se trataba, tal vez, de Mattia Gasparini-, y cuyos muros iban a cubrirse con «pekín de Lucca» sobre el cual no hay más noticias. Pero en cambio sí las hay sobre otras compras de telas chinescas, traídas directamente por la Compañía de Suecia o adquiridas a comerciantes madrileños, que tuvieron un gran

${ }^{24}$ «Por orden del exmo. sr. marqués Squilace, a D. José Gil de la Torre, director del almacén general de tejidos de las Rs. Fábricas de Talavera, su fecha 19 de febrero de este año, reglado al gusto y disposición del exmo. Sr. Conde de Gazola, se han ejecutado en dichas fábricas las estofas de seda, y con oro, que se han entregado para el Real Cuarto de la Princesa $\mathrm{N}^{\mathrm{a}} \mathrm{S}^{\mathrm{a}}$ en el Real Palacio de esta Villa:

Primeramente se pusieron en poder del sr. D. Francisco Sabatini, 49 paños de grodetur color de plata, matizado en doble espolinado, y a doble liage, con 269 varas y media para vestir las paredes del real cuarto de besamanos de la prince-

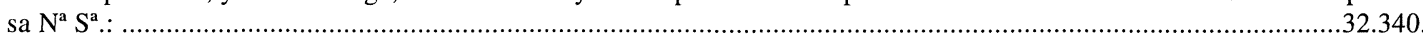

Id., se entregaron a dicho señor dos paños con 13 varas y cuarta

Asimismo se entregaron en pedazos, cinco: ........................................................................................

Ciento noventa varas y diez dozavos de grodetur color lila fino con oro, en diferentes hilados, y con matices de seda, uno

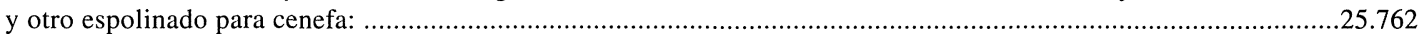

Treinta y siete flores de oro grandes en glasé, axula (?) y torzal, puestas en campo de grodetur lila fini de media vara de

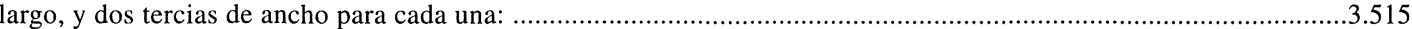

Asimismo se han entregado por disposición de dicho señor Sabatini a D. Andrés Jiménez camero, para las cortinas del citado Real Cuarto, cuarenta y ocho paños del mismo grodetur matizado con 321 varas y $7 / 24:$...........................38.555

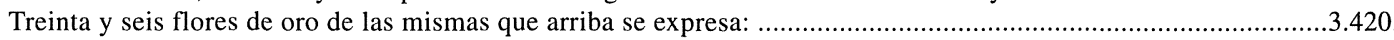

Ciento ochenta y ocho varas, y 14/24 de grodetur lila fino con oro y matices como el de arriba para cenefa de dichas

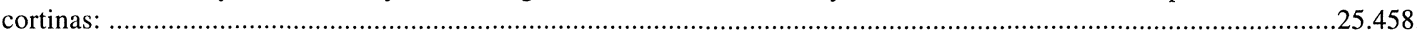

Seis varas y media de dicho grodetur color de plata matizado para mampara de una chimenea: ................................780.

Cuatrocientas sesenta y nueve varas de tafetán doble blanco para cortinas de verano del citado R1. Cuarto:............7.504.

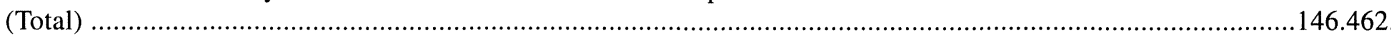

${ }^{25}$ Gazola a Squilace, 23.2.1766, sobre el pago de los géneros de Talavera. Adjunta la cuenta. 6.4.1766, Montealegre a Sabatini, cuentas de los cameros y mercaderes de seda por los géneros para el cuarto de los príncipes en Palacio. 13.5.1766, Gazola a Muzquiz, sobre el pago de los géneros de Talavera. 25.5.1766, R.O. a Tesorería General para que se paguen a la Fábrica de Talavera 146.462 rs. por las estofas de seda y oro entregadas a Gazola para las colgaduras, y otros adornos del cuarto de la Princesa.

Paralelamente se llevó a cabo similar operación en los cuartos de los príncipes en los restantes palacios, como el de Aranjuez; cfr. 21.1.1766, Montealegre a Sabatini 
peso en la decoración de los aposentos privados de Carlos III y sus hijos, en especial como colgaduras de verano, y acerca de las cuales se habían tomado decisiones en fecha muy temprana. A principios de 1762 ya se había decidido que los dormitorios del rey y de la reina y sus gabinetes respectivos, así como «la cama a la imperial» del rey serían de pekín. Gazola, como siempre, estuvo encargado de disponerlo, y a principios de marzo de 1762 envió a Squilace las medidas correspondientes. Estas eran tanto las de las colgaduras, es decir las de las habitaciones mismas, proporcionadas por Sabatini ${ }^{26}$, como las de las camas ${ }^{27}$. En ambos cómputos intervino el tapicero del rey Edmond le Goujeux. Las especificaciones sobre las camas son sumamente detalladas, permitiendo imaginar perfectamente su disposición ${ }^{28}$, y es destacable la insistencia de Gazzola en la correspondencia entre las colgaduras de las paredes y de la

${ }^{26}$ «Memorie delle misure de’ parati cinesi o siano pekin per alcune stanze degli appartamenti della Maestà del Rè, e della Regina nel nuovo Rl. Palazzo.

Per la stanza da letto di S.M. il Re, teli n. ${ }^{o} 32$ di lunghezza ogni telo, piedi 16 polsi 3 di Parigi.

Per un gabinetto del Rè fra la stanza dodetta da letto, ed un gabinetto della Regina teli n. ${ }^{\circ} 15$ di lunghezza ogni telo, piedi 13 polsi 10 di Parigi.

Per la stanza da letto della Regina teli $n .^{\circ} 30$ di lunghezza ogni telo piedi 16, polsi 3 di Parigi.

Per il gabinetto che deve servire di toilette della Regina teli n. ${ }^{\circ} 20$ di lunghezza ogni telo piedi 13 , polsi 10 di Parigi.

Nota: quando si vogliano da S.M. li leti corrispondenti le sod. misure de la lunghezza de parati ne dovranno lo notare, avenendo chi li colori de parati per il fondo dovranno essere diversi.»

27 «Cuenta del raso che necesita para la parato las cortinas delle porte e ventana e la cama de S.M. en el Palacio Nuevo. Primeramente necesita para toda la colgadura con los sopraporta e ventana, vara 540.

Para las cortinas dellas portas e vantanas necesita vara 270 .

Para la cama necesita vara 330.

Para los sitiales che potrano necesitare come canapé, sedie e taburetes in circa vara 60 .

Che asse en todo vara castelanna 1.200.

Las medidas tomadas da io Edmond Le Gougeux».

${ }^{28}$ Gazola a Squilace, 3 de marzo de 1762.

Medidas de la cantidad de pekinés necesario para Palacio, 1762

«Pieces de Pekin necessaires pour les appartements du Roy, et de la Reyne au nouveau Palais Royalle.

32 toiles de la longeur de 16 pieds et 3 pouces de Paris pour la chambre du lit du Roy.

15 idem de la longeur de 13 p. et 10 p. idem pour un cabinet du roy, et autant pour un autre de la Reyne.

30 idem de la longeur de 16 p. et 3 p. pour la chambre du lit de la Reyne.

20 idem de la longeur de 13 p. et 10 p. pour la toilette de la Reyne.

A Madrid ce 2 mars $1762 »$.

«Mesures des pieces necessaires pour un lit a l'Imperiale de Pekin des Indes, mesurées au pied de Roy. 2 rideaux de 16 piedas 6 pouces de hauteur sur 14 pieds de largeur. 4 bonnes graces de 16 p. 6 p. de hauteur sur 4 p. 6 p. de largeur 1 dossier de 14 p. de hauteur sur 5 p. 9 p. de largeur 1 chantourné de 6 p. de hauteur sur le milieu et environ 4 p. 4 p. sur les coins et de largeur 5 p. 9 p. Pour le tour de limperiale 4 pieces, 2 de 7 p. 6 p. de longeur sur un pied 4 p. de largeur, et les deux autres de 5 p. 9 p. de longeur sur un pied 4p. Le fond de l'Imperiale 6 pieds 3 p. de longeur sur 4 p. 6 p. de largeur 4 Petites pentes en dedans delImperiale deux de 7 pieds 6 p. de longeur sur 1 pied 5 p. de hauteur, deux autres de 5 pieds 9 p. de longeur sur un pied 5 p. de hauteur. 3 grandes pentes en dehors de l'Imperiale, deux de 8 p. 3 p. de longeur, sur deux pieds 6 p. de hauteur, et une de 6 p. 6 p. de longeur sur 2 p. 6 p. de hauteur. Nota: les petites pentes auront plus de largeur de chaque coté pour en bas, un doigt, et les grandes d un doigt et demi pour en bas, c est ce qui s'apelle les regets.

Pour le courtepointe la longeur du carre 6 pieds 6 p., 2 pieds pour le traversin, 2 pieds pour le soubassement, en tout 10 pieds 6 p. de longeur. Largeur sur le carre, 5 pieds 9 p. Les deux soubassements de cote de 2 pieds d'hauteur chacun qui fait en tout de la largeur de la courteponte 9 pieds 9 pouces, et les deux soubassements fairont de 7 pieds 6 p. de longeur

Trois soubassements d'enbas 2 de 7 pieds 8 p. de longeur sur un pied 7 p. de hauteur, et un de 6 pieds de longeur sur un pied 7 p. de hauteur. Il faut qu'il soit plus long pour en bas d'une pouce de chaque cotepour les regets. Deux fresses pour tenir les rideaux de trois pieds de longeur sur 5 doigts d hauteur Deux petits morceaux d environ un pied en carre pour le tour de la courtepointe.

A Madrid ce 2 Mars 1762».

«Memoria delle misure de parati cinesi o siano pekin per alcune stanze degli apartamenti dell Maesta del Re, e della Regina nel nuovo R1. Palazzo.

Per la stanza da letto di S.M. il Re teli n 32 di lunghezza ogni telo, piedi 16 polsi 3 di Parigi.

Per un gabinetto del Re frala stanza sod(etta) da letto, ed un gabinetto della Regina teli n 15 di lunghezza ogni telo piedi 13 polsi 10 di Parigi.

Per la stanza di letto della Regina teli n 30 di lunghezza ogni telo piedi 16, polsi 3 di Parigi.

Per il gabinetto che deve servire di toilette dell Regina teli n 20 di lunghezza ogni telo piedi 13 polsi 10 di Parigi.

Nota: Per quanto si vogliano da S.M. li letti corrispondenti, le sole misure de la lunghezza de parati ne daranno la norma, avertendo che li colori de parati per il fondo dovranno essere diversi $==\mathrm{G} .==$ » 
cama en cada cuarto, cuando envió las medidas de las telas para «... un letto per la Camera d'Estate del Rè perché ne possa ordinar due delle stesse misure acciò se ne abbino un altro per la Maestà della Regina; coll'avvertenza di prescrivere che siano ognuno dello stesso colore respetivamente delle stanze per le quali devono essere destinati. Prego pure V.E. di umiliare a S.M. che il picciolo stipetto che mi ha comandato spero presentarselo domenica se il maestro non mi inganna...» Gazzola ampliaba, como vemos, sus competencias del ámbito textil al mobiliario ${ }^{29}$. Pocos días despues la Real Compañía de Suecia recibió el encargo de traer las de China: no llegaron hasta marzo de $1765^{30}$. Una interesante carta del mayordomo mayor a Sabatini proporciona curiosos datos sobre el sentido que Carlos III daba a la ornamentación de sus gabinetes y a la atención que prestaba a detalles como estas colgaduras chinescas: Sabatini había «reconocido con D. Francisco García de Echaburu las colgaduras chinescas, que últimamente vinieron de Cádiz, y el modo en que ambos las han destinado; y S.M. se ha dignado conformarse en el todo de la distribución de ellas, a excepción de la de fondo color de rosa, y manojos de flores al natural dispuesta ponerla en el Gabinete de medio, en donde está la chimenea del mismo cuarto de S.M., pues esta, según cree, vino determinadamente señalada para el gabinete en que han de ponerse las pinturas de David Teniers, y de Bruguel, aunque duda S.M. si es esta, o la de fondo azul, y en tal caso quiere S.M. que se reserve, y no se eche mano de la de color de rosa, y que en su lugar puede ponerse, si pareciese, la de fondo blanco, pues no conviene en que, si no conforman las medidas del gabinete de medio con las varas de la tela, por ser mayor, o menor, se haga pedazos; y V.S. dígame cuál de las dos colgaduras entre esta de color de rosa, y la azul, es más conforme en las medidas a la pieza de las pinturas, para hacerlo presente a S.M. y esperar su Rl. Resolución» ${ }^{31}$.

Por otra parte, Squilace disponía en Madrid de un italiano - ingeniero, al parecer- especialista en pintar «chinerías» en telas de esta especie, como era costumbre en occidente: a este efecto Gazola había encargado «pekin de Lucca», o sea tafetán de seda para pintar ${ }^{32}$.

\footnotetext{
${ }^{29}$ Gazzola a Squilace, 3.3.1762.

${ }^{30}$ El encargo se había hecho el 7 de marzo de 1762 a través del cónsul de Suecia barón de Hildebrand, en virtud de una sugerencia hecha a Squilace por Gazola el 3 de marzo. El 27 de septiembre de 1763 un agente de la misma compañia, C.F. de Heland, expone a Squilace que «los géneros... no han podido, según la Compañía deseaba, venir en los últimos navíos que han vuelto, tanto a causa de la sazón que no estaba buena para los tintes, como a causa de no perder el tiempo favorable para su navegación, pero quedaron otros dos navíos... que volverán el próximo año 1764...» Se les esperaba en Cádiz a principios de Octubre de 1764, pero no llegaron hasta el 5 de marzo de 1765 . Su coste ascendió a 630.000 pesos, y comprendía «un juego para cama imperial, dos para colgadura de dos piezas grandes, y dos otras para gabinetes que en 1762 se le encargó», como se avisaba el 24.9.1764 al administrador de la aduana de Cádiz. En detalle, era lo siguiente:

Pekinés traído de Oriente por la Compañía Sueca

«Razón de lo contenido en los dos cajones que con géneros de China han venido en el navío sueco nom los estados extranjeros, y se remiten con Manl. Santos a Madrid en 7 de Marzo de 1765 para entregarlos a la disposición del Exmo. Sr. marqués de Squilace.

N 1 MT Contiene 10 piezas de color amarillo con pajaritos 3 dhas. azules con conchas y flores 4 dhas color de rosa con macetas de flores 8 dhas verdes con frutas y flores 3 dhas blancas con ramazón

N 2 MTS Contiene

2 piezas que contienen dos buenas gracias 6 dhas para las cortinas 1 dha con tres pendientes para lo exterior de la Imperial 1 daha con tres cenefas para el trabesin de la cortapunta 1 cha el fondo de la imperial 2 dhas con dos pendientes para lo interior de la Imperial 1 dha. con tres rodapiés 1 pieza con cuatro pedazos para el claro de la Imperial 1 paquete con trenzas para sostener las cortinas y dos pedacitos para el claro de la cortapunta 1 dha con el dosel 1 dha. el cuadro de la cortapunta 1 dha con el contorno.»

${ }_{31}$ Montealegre a Sabatini, Aranjuez 20 de abril de 1765: «Señor mío: he dado cuenta al Rey de cuanto me expone V.S. en su carta de ayer ...». Los gabinetes mencionados eran los del despacho o de maderas finas, llamadas actualmente «del Rey Francisco».

${ }^{32}$ Gazola a Squilace, 30.4.1764: «Io ignoro ove si trovi quello che V.E. aveva destinato per dipingere alla chinese li cinque gabinetti del Rl. Palazzo per cui si ordinarono, e giunsero li pekin da Lucca, se piace a V.E. potrà ordinarli che si intenda meco a Segovia per consegnarli li teli corrispondenti acciò possa intraprenderne l'opera». Respuesta el 2 de mayo: «que el sujeto que ha de pintar los (muebles) de los cinco gabinetes se halla empleado en la obra del camino de Andalucía, y no pudiéndose apartar de él, puede enviar aqui las telas y medidas a fin de poner él mano».
} 
A la vez, Gazola y Sabatini adquirían otras piezas de pekín para los restantes tres gabinetes. El conde andaba disponiendo uno de ellos en mayo de 1764, y deja claro que para ello tomaba siempre consejo del arquitecto, y que ambos se atenían a la práctica francesa en decoraciones de esta especie ${ }^{33}$. Al año siguiente Sabatini compraba al comerciante madrileño Alfonso Ximénez quinientas varas de colgadura «de lo más especial chinesco que se haya visto... muy necesarias por la falta que tenemos en Palacio de colgaduras de verano» ${ }^{34}$. Un mes despues, siempre supeditado al parecer de Gazola en lo que a decoración textil se refiere, proponía una compra de tejidos chinescos en Madrid por ser insuficientes las que tenían, habiendo decidido el rey que no se colgasen donde estaba proyectado las que habían venido de China salvo una. «El señor conde de Gazola antes de salir de Madrid me entregó unas veinte y tres piezas de telas chinescas, cada una de diverso color, y labor, que son como muestras, pues cada una de ellas tiene otras doce a lo menos del mismo color, y labor, para aplicar algunas de ellas en colgaduras de verano en el Nuevo Real Palacio...» ${ }^{35}$. Precisaba luego «que exceptuando, según la orden de S.M., las que últimamente vinieron de Cádiz, tengo ya desde su principio - como era razón - aplicadas las que existían anteriormente, y las que compré por mi mano; tengo tambien empleados todos los mueres de aguas y damascos que anteriormente vinieron de Valencia, y fortuna que la Reina Madre no quiere colgaduras de verano en su cuarto, pues siendo el palacio bastantemente grande, bastantes telas eran de menester, y lo que teníamos no era una gran porción; por lo que he pasado a examinar la cantidad precisa de dichas telas de china que se necesita para colgaduras, supuesto que el rey no quiere se pongan sino pinturas en sus gabinetes - según me avisa el Sr. Mayordomo Mayor - se reduce a poner de estas telas, por la variedad, un gabinete al Sr. Infante D. Luis, otro a los Sres. Infantes, otro a la Princesa, y el dormitorio con colgadura y cama a la Sra. infanta $\mathrm{D}^{\mathrm{a}} \mathrm{M}^{\mathrm{a}}$ Josefa; para lo cual, en lugar de las seis muestras que propuse a V.E. he escogido tres, una muestra tira 288 varas; otra de 262 , y otra de 378 , que todas juntas hacen 928 varas, que bastan para el mencionado fin, cuando no se quieran poner otras telas o colgaduras, buscándolas hechas o de Valencia, o de

\footnotetext{
33 Gazola a Squilace, 5 de mayo de 1764. Eccellenza. Fortunatamente avendo incontrata l'occasione che passa costì il console Generale Britanico, rimeto a V.E. come mi ha comandato il pekin correspondente ad un gabinetto del Rl. Palazzo Novo acció V.E. possa impiegare l'abilitá della persona destinata a dipingerlo.

Conveni tenga questi presente che li teli devono esser ventiuno, e questi di cinque barre ciascheduno di lunghezza, e per ciò si mandano barre 105 essendo il pekin segnato da una parte per una lunghezza con un picciol tagli di forbice per maggior chiarezza acciò non segua equivoco nel taglio delle parte, o teli che devono formare l'apparato del gabinetto. Avvertendo al diletante che alle due estremità cioé a dire verso il cornicione, e verso il lambris dovrà lasciare come al incirca cinque dita di liscio, o non dipinto, affinche nell'adattare li teli si lasci luogo per gli astragalli o siano cornici dorate all'intorno che ora costumami larghe assai come lo comandano li signori francesi.

Quando saranno terminati, o prossima a terminarsi detti teli si parlerà di sopraporte, portier e tendine dello stesso pekin per regolarli secondo il genio, ed il metodo che auri dipinto il resto.

Desidero d'essermi spiegato a dovere acciò non succedono inconvenienti, ma mi lusingo che non ci sarà che terche il tutto l'ho fatto colla direzzione e consigli del sig. Sabatini...»

${ }^{34}$ Sabatini a Squilace, 7 de abril de 1765. Costó cincuenta mil reales.

${ }^{35}$ Sabatini a Squilace, 5 de mayo de 1765 . «... y habiendo reconocido los cuartos se puede poner de ellas en un gabinete del Rey, en otro del sr. Infante D. Luis, en otro de los Sres. Infantes, en el dormitorio y tocador de la Sra. Infanta $\mathrm{D}^{\mathrm{a}} \mathrm{M}^{\mathrm{a}}$ Josefa, en los gabinetes de la Sra. Princesa; y a este fin he escogido unas seis muestras, que con sus piezas correspondientes completan la cantidad que tenemos de menester, y por el motivo de hallarse el mencionado sr. Condde fuera de Madrid he tratado con el interesado, y pide a 64 rs. la vara por ellas; V.E. enterado de todo resolverá, y mandará cuanto más antes se pueda lo que tengo que hacer.

Es menester también que sepa que de las cinco colgaduras chinescas que últimamente vinieron de Cádiz, aplica solamente una en su dormitorio; pues no quiere el Rey que se apliquen las otras cuatro por ahora, por la razón que no teniendo ... las piezas en donde han de servir, no basta la colgadura, y el rey no quiere que se apliquen en otros cuartos conforme me lo manda el sr. mayordomo mayor de parte de S.M.»

Resuelto por Squilace el 9 de mayo: «que antes de entrar en la compra de las colgaduras de China que propone, es menester que exceptuando, según la orden del rei, el resto de las que vinieron de Cádiz, junte las que existían con las compradas por su mano, tome sus medidas para emplear estas con preferencia, y pasando sucesivamente a ver las demás que se necesitan para colgar los gabinetes que dice, avise la cantidad precisa, y el precio último en que el mercader pretenda, en el concepto de que hallo muy caro el de 64 rs. la vara que pide».
} 
Talavera. he tratado con el sujeto que tiene de venta dichas telas, y dice que no las puede dar a menos de 56 reales la vara...» ${ }^{36}$. Es bien digna de subrayarse la preferencia de Isabel de Farnesio por colgar los cuadros sobre las paredes sin tela.

Puestos a comprar telas de China a mercaderes diversos, el mayordomo mayor y el arquitecto acudieron a comerciantes de Cádiz en 1765, cuando fue necesario más de este género para el gabinete del rey. Poco despues hubieron de colgarse de pekín también los gabinetes y dormitorio de infantes, infanta y futura Princesa ${ }^{37}$.

\section{Epílogo}

Estrenado Palacio con la mayor parte de sus colgaduras definitivas, y perfeccionadas las que faltaban para la boda de los Príncipes, Gazzola pudo sentirse orgulloso de su tarea como director de los textiles, que había llevado a puerto con la eficaz colaboración de su superior, Squilace, y de su protegido, Sabatini. Pero no todo marchaba como la seda, en aquella sazón. Ese bien trabado equipo de italianos, a quienes es preciso atribuir colegiadamente el trato tan distinto que recibieron Lyon y Valencia en el encargo regio de colgaduras, quedaba roto el domingo de Ramos de 1766, con el estallido del Motín que se conoce desde entonces con el nombre del ministro entonces caído. Con su sucesor, Múzquiz, Gazzola hubo de dirimir poco después el pago de los trabajos ya acabados, en cuya supervisión económica nunca había querido entrar: «El rey podrá contestar a V.S. que en Nápoles se dignó emplearme en muchas dependencias de esta naturaleza... pero logré siempre la satisfacción de no ingerirme nunca en intereses por no ser correlativos a mi genio, y talento...» Squilace había llevado siempre la gestión de las sedas, e incluso entraba a veces en la aprobación de los diseños, como hemos visto, pero ante su caída, el lunes Santo, despachó a Gazzola — según éste mismo cuenta«con la expresión de no ser de su inspección el allanar la cuenta: que yo la ajustase y avisase el importe. Bien ve V.S. que en aquel tiempo no me hallaba en proporción de pensar a la ejecución de tal orden, ni el sr. Squilace en caso de oir mis razones en contrario» ${ }^{38}$. En efecto, aquel día Squilace oía demasiadas cosas, mientras Gazzola proponía al rey ordenar una ejecución bien distinta, la de los madrileños sublevados, con metralla. Carlos III demostró por esta vez un tacto más sedoso que el director de su Real Colegio de Artillería. En su disgusto hacia el indisciplinado Madrid pensó, sin embargo, en trasladar la corte a Valencia, esa ciudad industriosa en medio de una huerta rica, tal como permitían imaginársela las flamantes colgaduras de su palacio ${ }^{39}$.

Madrid, por supuesto, continuó siendo la sede de la Corte; y en el Palacio Real las colgaduras no cambiaron mucho durante el resto del reinado, como permite comprobar el inventario llamado de Carlos III, que en realidad recoge el estado hacia 1793, después de los considerables cambios emprendidos por Carlos IV cuando accedió al trono. La especial atención de este monarca a las artes decorativas, y en particular a las sederías, ha hecho que le prestásemos

\footnotetext{
${ }^{36}$ Sabatini a Squilace, 12 de mayo de 1765. Resuelto al día siguiente: «Que compre estos pequines, pero que sería bueno antes que hablare al conde Gazola para que también dijese su parecer». Como Sabatini dice, el rey no quiso más que pinturas en los gabinetes, y por tanto de las colgaduras traídas de China por la Compañía sueca «aplica solamente una a su dormitorio, pues no quiere el rey que se apliquen las otras cuatro por agora, por la razón que no teniendo las piezas en donde han de servir, no basta la colgadura, y el rey no quiere se apliquen en otros cuartos». Finalmente, tras consulta con Gazola, se compraron a Juan Lavedan 854 varas por 4.824 reales.

37 Squilace a Sabatini, 9.5.1765, compra de telas de china para los gabinetes y dormitorio de infantes, infanta y futura Princesa. Id., 13.5.1765.

${ }^{38}$ Gazola a Muzquiz, 21.5.1766, sobre el pago de los géneros a la Fábrica de Talavera, hechos para la colgadura de la antecámara de la Princesa. Le habla de su carta a Squilace del 23.2.1766.

${ }^{39}$ Fernán-Núñez, Conde de: Vida de Carlos III.
} 
menos a la mostrada por su padre. Sin embargo, Carlos III, al disponer el amueblamiento de su residencia, mostró hacia los tejidos un cuidado notable, que es preciso considerar precedente del desarrollado por su hijo en este campo; el trato que los sederos lioneses recibieron de padre e hijo fue bien diferente, empero; $y$, en cuanto a sus respectivas actitudes hacia la industria valenciana, sin entrar en valoraciones acerca de la de Carlos IV es preciso destacar el valor del proteccionismo que Carlos III y Squilace desplegaron en su favor, y que debe ser entendido no sólo desde su alcance económico, sino simbólico, pues lo tiene el que las paredes del Palacio del Rey no estén tapizadas con telas francesas; hecho más conceptual que visible, pues los cuadros de la riquísima colección real tapizaban de arriba abajo, sin dejar resquicio, las paredes forradas de seda. En su papel de «basso continuo», aquellas telas formaban parte de una imagen que es preciso reconstruir, aunque sólo idealmente, pues el Palacio ha adquirido nuevas y respetables apariencias durante los dos siglos siguientes de habitación y vida ${ }^{40}$. pero su sentido quizá lució como nunca cuando se engalanó para su estreno.

\section{APÉNDICE DOCUMENTAL}

Muestras enviadas por Caillat y Chirac a Madrid en 1763

«Lyon le 6 Avril 1763. [En esta lista, el primer número es el que corresponde al patrón; y a continuación se expresa su ancho, que es una fracción de ana, o aune, medida de tapicería]

Patron $\mathrm{n}^{\circ} 1$ - Echantillon en taffetas chiné qui ne doit servir que pour voir l'effet du dessein, on peut faire des fonds en toutes couleurs qui reussissent toutes bien mais principalement dans le fond blanc pur; prix: 8.10 .

N..$^{\circ}$ - Autre dessein nouveau en $1 / 2$ aune de large, prix: 6.15

N. ${ }^{\circ}$ - Autre id: 6.15 .

Estos tres «pliés dans un papier»

N. ${ }^{\circ} 15-$ Cotté une aune damas cramoisi fin liseré vert et blanc ou couleur d'or, 22.

$\mathrm{N} .^{\circ} 1$ - Un rideau de moire soye blanche portant 3 aunes de hauteur sur 3 let. de largeur de 1/2 aune de large peint. L'aune coutera 25.

$\mathrm{Si}$ on le désire sur une moire tramée fil telle que l'echantillon $\mathrm{n} .^{\circ} 2,21$.

N. ${ }^{\circ}$ - $1 \& 1 / 2$ aune echantillon de moire tramee fil, 18

et en moire toute soye, 22

[Para estos dos, al margen]: dessein à grosses roses et à peau de tigre

N. ${ }^{\circ}$ 3- 3/4 echantillon de moire soye de la largeur de 11/24 dont le fond est peint en petit jaune, 23.

L'on peut faire peindre sur de la moir, sur soye, dans quels fonds de couleur que l'on voudra soit bleu, lilas, jaune, rose, et vert, ces couleurs ne passeront point et seront plus parfaites qu'a ce bout d'echantillon que l'on a ete forcé de faire à la hâte.

N. ${ }^{\circ}$ 4- 3/4 taffetas blanc en largeur de 7/12 peint tel quel, 19

Si on le veut sur de la moire soye largeur de 11/24, prix: 23.

N. ${ }^{\circ}$ 5- 3/4 taffetas blanc largeur 7/12, prix: 18.

[Al margen:] dessein à plumes

Ce dessein peut aussi se mettre sur de la moire soye en distribuant le dessein sur deux largeurs tel qu'est peint le rideau $\mathrm{n}^{\circ} 1$ ci-dessus, pour lors l'aune coutera 21 ; et en moire tramée sur fil, 17.

N. ${ }^{\circ}$ 6- Une hauteur de meuble en carrelé broché laine et soye trame fil portant $33 / 4$ aunes de haut sur deux lets formant 7 aunes l'aune coutera 30; et si on le desire broché en chenille, 60

[Al margen:] dessein aux iris et aux perles

N. ${ }^{\circ}$ 7- Une hauteur en gros de tour porcelaine portant 2 aunes $1 / 2$ de haut et de la largeur de $5 / 6$ executé tel que l'échantillon, l'aune coutera 72 .

[Al margen:] representant le temple de l'hymen

N. ${ }^{\circ} 8-3 / 4$ gros de tour meme dessein du n. ${ }^{\circ} 7$ destiné pour fauteuils, canapé et tabourets, 72

N..$^{\circ}$ 9- 3/8 carrelé verd broché soye, cette etoffe telle qu'elle est doit être placée dans un salon ou chambre qui soit distribuée par paneau, c'est à dire que l'on peut placer un paneau d-etoffe qui est dans toute sa largeur et un paneau de glace ou un paneau de boisage orné de sculpture, 74

N. ${ }^{\circ} 10-1 / 2$ aune carrelé verd broché soye destiné pour lit et fauteuil du dessein du n. ${ }^{\circ} 9,72$

${ }^{40}$ Cfr. Pilar Benito García, «Los textiles y el mobiliario en el Palacio Real de Madrid», Reales Sitios, n. ${ }^{\circ} 109$. 
De tous les desseins, l'on fait exprés les fauteuils, canapés et automanes [sic] et cordons de sonnette, en envoyant les patrons de fauteuils qui seront en toile ou en papier, il faut que le tapissier y mette l'emplissage avant que de couper les patrons.

N. ${ }^{\circ} 11$ Le dessein peint sur papier blanc qui represente une corbeille en chiné doit etre une fois et demi plus grand pour aller sur du taffetas 7/12; prix, 14 .

Dans un petit paquet long en toile cirée: Esquisse d'un taffetas chiné de la largeur de 5/8 d'aune. Le dessein rendra exactement sur l'étoffe tel qu'il est peint quant aux grandeurs des sujets et aux nuances, à la difference près qu'il sera plus agréable encore en etoffe, la peinture du papier n'étant que croquée. Si on a quelques changements à y faire on tachera de s'y conformer. On verra les colonnes verd d'eau peintes en trois manières ainsi que trois ou quatre sortes de pannaches, et d iris, c est pour faire le choix qui conviendra; prix, 13.10. On est prié de renvoyer incessamment cet esquisse ainsi que tous les echantillons contenus au present memoire».

«Note des echantillons partis le 13 Avril 1763

Un paquet $n .^{\circ} 1$, Cotté A

N. ${ }^{\circ}$ 1: Etoffe à ramage de rose fond blanc des indes destinée à faire le plein de la tapisserie, du lit des rideaux, des bonnes graces, dossier, courtepointe, et generalemment tous les pleins des petits meubles, comme fauteuils, canapés, ecrans, paravets etc.

N. ${ }^{\circ} 1$ Etoffe à deux grandes guirlandes de fleurs nué sur fond olive pour etre decoupées appliquées sur un fond blanc des indes, dans differentes formes de festons, et employée dans les bonnes graces, les pentes et soubassemts du lit, et former une grande bordure à colonne torse ou autrement pour encadrer la tapisserie et les portieres de l'appartement.

N. ${ }^{\circ} 1$ Etoffe à 4 petits bordures sur fond blanc des indes en feuille de chene enveloppé d-un petit ruban nué rose pour servir à encadrer generalement tous les petits meubles, et à former la grande bordure avec les guirlandes decoupées de dessus l'etoffe olive cy dessus.

Un paquet $n .^{\circ} 2$, Cotté $B$

N. ${ }^{\circ} 2$ Cote B, N 2: Etoffe à grand bouquet de fleurs naturelles nué sur fond blanc des indes pour former le plein de tous les meubles en general comme letoffe cy dessus destinée pour le fond plein du n 1 .

N. ${ }^{\circ} 2$ Etoffe à deux grandes bordures en forme de treillege nué, pour encadrer la tapisserie, les portieres, les bonnes graces du lit, et former les pentes et soubassement en les decoupants.

N. ${ }^{\circ} 2$ Etoffe à 4 petites bordures analogues à la grande pour encadrer géneralement tous les petits meubles, comme fauteuils, etc.

Un paquet n. ${ }^{\circ}$, Cotté $\mathrm{C}$

N. ${ }^{\circ}$ : Etoffe à ramage de volubilis fond blanc des indes destiné pour le fond plein de tous les meubles en general.

N. 3 Etoffe à deux grandes bordures et deux petites sur la largeur nué camayeu bleu et girofe sur un fond verd pour encadrer la tapisserie, les portieres, bonnes graces, et former les pentes et soubassement du lit

$\mathrm{N}^{\circ} 3$ Etoffe à 4 petites bordures sur la largeur fond verd, nué camayeu verd pour etre decoupé et servir à former la jonction de la grande bordure fond verd sur le fond blanc à ramage de vlubilis et à encadrer tous les petits meubles.

Un paquet $n .^{\circ} 4$, cotté $\mathrm{D}$

N. ${ }^{\circ} 4$ Etoffe à ramage de chevrefeuil fond blanc des indes destinée pour le fond plein de tous les meubles en general

N. ${ }^{\circ} 4$ Etoffe à 4 bordures sur la largeur en feuille d-olivier nué camayeu verd pur encadrer tous les petits meubles.

La grande bordure du n. ${ }^{\circ} 3$ nué camayeu bleu et girofle sur fond verd

peut servir egalement à ce meuble et fera tres bien. Le prix de toutes les etoffes cy-dessus sera de 34 à 36 les unes dans les autres.

N. ${ }^{\circ}$, aunes 1 1/2: Echantillon d-un velours fond cramoisi verd et or, dont le meuble peut se faire en fond tout soye, tel que celuy du n 5. Si le dessein plait, on peut le faire dans les couleurs que l'on désirera, le prix en deux couleurs sera 42 ., et en une seule couleur comme bleu, verd jaune etc. sera de 37.

$\mathrm{N} .^{\circ} 2$, aunes $11 / 2$ Echantillon d'un velours en dorure qui reussira tres bien en tout soye, il y aura quelque petite correction à la grande fleur du milieu qui rendra le dessein plus agreable, ce meme dessein est destine pour les dossiers de fauteuil, car on travaille à un dessein en grand et analogue pour la tapisserie, le prix en trois couleurs sera 48 .

N. 3 , aunes $1 / 2$ Echantillon d'un velours fond or cramoisi et blanc du meme dessein du n 1, qui ne doit servir que pour en voir la couleur qui flatte davantage.

N. ${ }^{\circ} 4$ aunes $1 / 2$ Echantillon d'un velours à ramage en une seule couleur qui reussit tres bien auquel est joint un petit echantillon de velours cerise et blanc pour donner une idée de la couleur, le prix en couleur ordinaire sera 34. 
N. 5 aunes 1/4 Echantillon d'un velours à bande de tigre et marbre fond satin blanc, si le dessein qui imite la fourrure étoit agrée, on y mettroit des bouquets en fleurs naturelles beaucoup plus marquants; 48 . N. ${ }^{\circ}$ 6 aunes 1 Echantillon d'un damas en 3 couleurs qui ne doit servir que pour donner une idée d'un velours en 4 couleurs du meme dessein exactement qui rendra tres bien, et dont le velour sera en fond satin blanc comme le n 5, et sera varié de 4 couleurs differents; 52 .

Sans numeros,

aunes 1 1/6 Echantillon d un taffetas chiné fond blanc, 12.

aunes 1 a/6 Autre $d$ un meme fond jaune, 12 .

Deux desseins sur un rouleau: Celui à draperie avec une corbeille de fleurs sera en fond satin et gros de tours ou damas, la draperie sera brochée en soye le prix environ sera 50.

$\mathrm{Si}$ on souhaite la draperie en chenille cela fera une augmentation de 12 à 13 l'aune, et si on veut le fond en couleur fine encore une augmentation de 6 l'aune. Celui à paysage sera dans les memes fonds de celui cy dessus et le prix en couleur ordinaire sera 53.

On prie de renvoyer le tout le plus promptement possible apes que l examen en aura été fait».

«N. 3 : Echantillon dune etoffe fond jonquille cannellé de $2 / 3$ de large dont le prix est de 150 l'aune.

L'on fera les fonds et ciel de lit d'un seul dessein dans le meme goût de même que les sophas fauteuils et autres avec leus bordures, comme aussi la bordure pour l'encadrement de la tapisserie en envoyant exactement les mesures. Ces derniers objets augmenteront le prix de l'étoffe en proportion des frais qu'on sera obligé de faire pour les monter sur les métiers.

N. 3 On observera que dans cet echantillon les armes d'Espagne y sont en petit. Si ce meuble convient, alors on les mettroit plus en grand dans les coins de la tapisserie complette soit en broderie d'or et d'argent ou d'autre façon comme on le souhaitera.

Un autre petit echantillon d'environ 60 l'aune». 